\title{
INFLUÊNCIA DA TEMPERATURA DE ARMAZENAMENTO NA QUALIDADE PÓS-COLHEITA DE GOIABAS SERRANAS ${ }^{1}$
}

\author{
ALINE CRISTINA VELHO², CASSANDRO VIDAL TALAMINI DO AMARANTE ${ }^{3}$, \\ LUIZ CARLOS ARGENTA ${ }^{4}$, CRISTIANO ANDRÉ STEFFENS ${ }^{5}$
}

RESUMO - O presente estudo teve por objetivo avaliar o potencial de armazenagem de goiabas serranas em temperaturas de $23{ }^{\circ} \mathrm{Ce} 4{ }^{\circ} \mathrm{C}$. Os frutos foram colhidos em pomar comercial, localizado no município de São Joaquim-SC, e armazenados nas temperaturas de $23 \pm 1{ }^{\circ} \mathrm{C}\left(75 \pm 5 \%\right.$ UR, durante quinze dias) e $4 \pm 1{ }^{\circ} \mathrm{C}$ ( $90 \pm 5 \%$ UR, durante quatro semanas). Durante o armazenamento, foram feitas avaliações de respiração, produção de etileno, teor de sólidos solúveis (SS), acidez titulável (AT), cor da epiderme (ângulo hue; $h^{\circ}$ ) e ocorrência de escurecimento da polpa, manchas escuras na epiderme e podridões. Durante o armazenamento a $23{ }^{\circ} \mathrm{C}$, os frutos exibiram um padrão climatérico, com pico respiratório entre o $4^{\circ}$ e o $5^{\circ}$ dia. Nos frutos armazenados a $4{ }^{\circ} \mathrm{C}$, houve redução na respiração e na produção de etileno. Em frutos a $23{ }^{\circ} \mathrm{C}$, foi mais acentuada a redução nos valores de SS, AT e $h^{\circ}$ da epiderme, e aumento na incidência de manchas escuras na epiderme e de podridões, em relação aos frutos armazenados a $4{ }^{\circ} \mathrm{C}$. O armazenamento a $4{ }^{\circ} \mathrm{C}$ mostrou-se eficiente na redução do metabolismo respiratório e de produção de etileno, na preservação dos atributos de qualidade (SS, AT e $h^{o}$ da epiderme) e na diminuição da severidade das manchas na epiderme e podridões sem, contudo, evitar o desenvolvimento de escurecimento da polpa. O potencial de armazenagem de goiabas serranas a $23{ }^{\circ} \mathrm{C}$ é inferior a uma semana e limitado principalmente pelo desenvolvimento de distúrbios de escurecimento da polpa, manchas na epiderme, bem como podridões.

Termos para indexação: Acca sellowiana (Berg.) Burret, distúrbio fisiológico, podridão, respiração, etileno, amadurecimento, refrigeração.

\section{INFLUENCE OF STORAGE TEMPERATURE ON POSTHARVEST QUALITY OF FEIJOAS}

\begin{abstract}
This study was carried out to evaluate the storage potential of feijoas at temperatures of $23^{\circ} \mathrm{C}$ and $4^{\circ} \mathrm{C}$. Fruit were harvested in a commercial orchard in São Joaquim, Santa Catarina (SC), Brazil, and stored at $23 \pm 1{ }^{\circ} \mathrm{C}(75 \pm 5 \% \mathrm{RH}$, for 15 days $)$ and $4 \pm 1^{\circ} \mathrm{C}(90 \pm 5 \% \mathrm{RH}$, for 4 weeks $)$. Fruit were assessed during storage in terms of respiration and ethylene production rates, soluble solids content (SSC), titratable acidity (TA), skin color (hue angle; $h^{\circ}$ ), and incidence of flesh and skin browning, and rots. Fruit stored at $23^{\circ} \mathrm{C}$ exhibited a climacteric, reaching a peak of respiration rates between the $4^{\text {th }}$ and $5^{\text {th }}$ days. Fruits stored at $4^{\circ} \mathrm{C}$ had lower respiration and ethylene production rates. Fruit stored at $23^{\circ} \mathrm{C}$ had more substantial decreases of SSC, TA, and $h^{\circ}$ of the skin, and increases of skin browning and decay incidence, compared to fruit stored at $4^{\circ} \mathrm{C}$. The storage at $4^{\circ} \mathrm{C}$ was more effective to reduce respiration and ethylene production, to preserve the quality attributes (SSC, TA, and $h^{\circ}$ of the skin), and to reduce the severity of skin browning and decay, without avoiding the development of flesh browning. The storage potential of feijoas at $23^{\circ} \mathrm{C}$ is less than one week as a result of flesh browning and skin browning development and decay.

Index terms: Acca sellowiana (Berg.) Burret; physiological disorder; decay; respiration; ethylene; ripening; refrigeration.
\end{abstract}

\footnotetext{
'(Trabalho 007-10). Recebido em: 04-01-2010. Aceito para publicação em: 26-07-2010.

${ }^{2}$ Aluna do Curso de Mestrado em Produção Vegetal, Universidade do Estado de Santa Catarina (UDESC), Centro de Ciências Agroveterinárias (CAV). Av. Luiz de Camões, 2090, CEP 88520-000. Lages-SC. E-mail: alinecristinav@hotmail.com

${ }^{3}$ Ph.D., Bolsista de Produtividade em Pesquisa do CNPq. Professor do Departamento de Agronomia, CAV/UDESC, Lages-SC. Autor para correspondência. E-mail: amarante@cav.udesc.br

${ }^{4}$ Dr., Pesquisador da Empresa de Pesquisa Agropecuária e Extensão Rural de Santa Catarina (Epagri), Estação Experimental de Caçador. Rua Abílio Franco, 1500, Bairro Bom Sucesso, C. P., 591, CEP 89500-000, Caçador-SC. E-mail: argenta@epagri.sc.gov.br

${ }^{5}$ Dr., Professor do Departamento de Agronomia, CAV/UDESC, Lages-SC. E-mail: steffens@cav.udesc.br
} 


\section{INTRODUÇÃO}

O Brasil é considerado um dos principais centros de diversidade genética de espécies frutíferas ainda pouco estudadas. O potencial de exploração comercial de algumas dessas espécies frutíferas nativas tem sido identificado nos últimos anos à medida que são desvendados atributos de qualidade de seus frutos sob ponto de vista de apreciação e propriedades nutracêuticas (WESTON, 2010).

A goiabeira serrana (Acca sellowiana, sinonímia Feijoa sellowiana) é uma espécie frutífera da família Myrtaceae, nativa do planalto meridional brasileiro e do Uruguai (DUCROQUET; HICKEL, 1991). A espécie é cultivada comercialmente em vários países, tais como França, Israel, Itália, Rússia, Colômbia, EUA e Nova Zelândia (THORP; BIELESKI, 2002). No Brasil, a goiabeira serrana encontra-se em processo de domesticação, ocorrendo em populações naturais no sub-bosque da Floresta Ombrófila Mista e em alguns pomares comerciais (QUADROS et al., 2008).

$\mathrm{O}$ fruto da goiabeira serrana apresenta alta qualidade organoléptica, com um sabor doce-acidulado e um excelente aroma. $\mathrm{O}$ fruto é fonte de vitaminas e minerais, apresenta atividade antibactericida, antioxidante e antialérgica, sendo que a presença de flavonoides auxilia na atividade imunológica, auxiliando no controle de processos inflamatórios (WESTON, 2010). Além do consumo in natura, os frutos podem ser processados e utilizados na produção de sucos, geleias, sorvetes e bebidas (THORP; BIELESKI, 2002).

A maturação da goiaba serrana na planta varia muito entre frutos, podendo a colheita durar um mês para uma mesma cultivar e um mesmo local (DUCROQUET; HICKEL, 1991). No ponto ideal para consumo, o fruto desprende-se facilmente do pedúnculo mediante o toque ("touch picking") (THORP; BIELESKI, 2002).

A goiaba serrana é um fruto climatérico e apresenta elevados valores de respiração, produção de etileno e acelerado amarelecimento da casca quando mantida sob temperaturas $\geq 20^{\circ} \mathrm{C}$ após a colheita (AMARANTE et al., 2008). Por outro lado, os frutos da goiabeira serrana também podem desenvolver danos por frio, especialmente quando armazenados sob temperaturas inferiores a $4{ }^{\circ} \mathrm{C}$ (THORP; BIELESKI, 2002). Os sintomas de danos por frio consistem no aparecimento de regiões escurecidas próximos ao pedúnculo dos frutos, seguidos de um escurecimento interno ao longo dos elementos vasculares e na polpa (THORP; BIELESKI, 2002).

O presente estudo teve como objetivo avaliar o potencial de armazenagem de goiabas serranas em temperaturas de 23 e $4^{\circ} \mathrm{C}$.

\section{MATERIAL E MÉTODOS}

Os frutos de goiabeira serrana foram colhidos em um pomar comercial, formado por uma mistura de clones, localizado no município de São JoaquimSC, em abril de 2008, no ponto de colheita comercial identificado pela facilidade do desprendimento da planta. No laboratório, os frutos foram selecionados pela uniformidade de tamanho, cor e ausência de defeitos, como má formação e danos mecânicos.

Os frutos foram armazenados nas temperaturas de $23 \pm 1{ }^{\circ} \mathrm{C}(75 \pm 5 \%$ UR, durante quinze dias) e $4 \pm 1{ }^{\circ} \mathrm{C}(90 \pm 5 \%$ UR, durante quatro semanas). Os frutos foram analisados quanto à respiração e produção de etileno, cor da epiderme, teor de sólidos solúveis (SS), acidez titulável (AT) e ocorrência de escurecimento da polpa, manchas escuras na epiderme e podridões. Nos frutos mantidos a $23{ }^{\circ} \mathrm{C}$, as avaliações de respiração e produção de etileno foram feitas diariamente, sendo os demais atributos de qualidade avaliados após $0 ; 3 ; 6 ; 12$ e 15 dias de armazenamento. Nos frutos mantidos a $4{ }^{\circ} \mathrm{C}$, todas as avaliações (respiração, produção de etileno e qualidade) foram feitas após $0 ; 2 ; 3$ e 4 semanas de armazenamento, mais $8 \mathrm{~h}$ a $23{ }^{\circ} \mathrm{C}$.

Para as análises de respiração e produção de etileno, amostras compostas de oito frutos foram colocadas em jarras de $4 \mathrm{~L}$, com fluxo constante de ar comprimido $\left(120 \mathrm{~mL} \mathrm{~min}^{-1}\right)$, livre de etileno, a $23{ }^{\circ} \mathrm{C}$, segundo metodologia descrita por Argenta et al. (2003). No decorrer das avaliações, frutos que apresentavam podridões e manchas escuras na epiderme foram removidos das jarras. No ar efluente, foram analisadas as concentrações de $\mathrm{CO}_{2}$ e etileno, por meio de um cromatógrafo a gás (Shimadzu 14B, Japão), equipado com metanador (Shimadzu, MTN1), detector de ionização de chama e coluna de aço inoxidável (de $0,6 \mathrm{~m}$ e diâmetro interno de $2 \mathrm{~mm}$ ), empacotada com Porapak Q, 80 a 100 mesh (Supelco, Bellefonte, EUA). As temperaturas do forno, detector, metanador e injetor foram de $45 ; 120 ; 300$ e $110^{\circ} \mathrm{C}$, respectivamente. Os fluxos de nitrogênio, hidrogênio e ar utilizados foram de $70 ; 30$ e $300 \mathrm{~mL}$ $\min ^{-1}$, respectivamente.

As avaliações da cor da epiderme (ângulo 'hue'; $h^{\circ}$ ) foram feitas com um colorímetro Minolta CR-300, na região equatorial, com duas leituras, em lados opostos do fruto.

Os valores de SS e AT foram determinados através de suco preparado com espremedor tipo Champion (Plastaket Mfg). O teor de SS ( ${ }^{\circ}$ Brix) 
foi medido usando-se um refratômetro digital com compensação automática de temperatura (Atago, Japão). A AT (\% de ácido cítrico) foi determinada utilizando-se de $5 \mathrm{~mL}$ do suco do fruto, diluídos em $20 \mathrm{~mL}$ de água destilada, sendo esta solução titulada com $\mathrm{NaOH}$ 0,1 $\mathrm{N}$ até pH 8,1, usando um titulador automático (Radiometer Analytical, Dinamarca).

A severidade de escurecimento da polpa, manchas escuras na epiderme e podridões dos frutos foram avaliadas atribuindo-se notas de 1 a 4 , pela análise visual, sendo 1-ausente, 2-inicial, 3-moderada e 4-severa, correspondendo a $0 \%, 1-30 \%, 31-60 \%$ e $61-100 \%$ da superfície do fruto com sintoma, respectivamente. Em frutos que apresentaram manchas escuras na epiderme, buscou-se isolar e identificar, junto ao laboratório de fitopatologia, o patógeno, que poderia estar associado com o desenvolvimento destes sintomas.

Os experimentos, nas duas temperaturas, seguiram o delineamento inteiramente casualizado, com quatro repetições, sendo cada repetição constituída por oito frutos. Os dados foram submetidos à análise de variância (ANOVA) e a teste de comparação de médias (teste LSD; $p<0,05$ ), utilizando o programa SAS. Os dados de severidade foram transformados para $(\mathrm{x}+10)^{1 / 2}$ antes de serem submetidos à ANOVA.

\section{RESULTADOS E DISCUSSÃO}

Durante o armazenamento a $23{ }^{\circ} \mathrm{C}$, foi possível observar que os frutos exibiram um padrão respiratório do tipo climatérico, caracterizado por um aumento na atividade respiratória, atingindo um pico entre o $4^{\mathrm{o}}$ e o $5^{\mathrm{o}}$ dia após a colheita, com valor de aproximadamente $2.900 \mu \mathrm{mol}$ de $\mathrm{CO}_{2} \mathrm{~kg}^{-1} \mathrm{~h}^{-1}$ (Figura 1A). Resultados similares foram obtidos por Amarante et al. (2008), mostrando alta atividade respiratória, quando comparada a outros frutos climatéricos. Bron et al. (2005), trabalhando com goiaba comum, cultivar Paluma, mantida na temperatura de $23{ }^{\circ} \mathrm{C}$, obtiveram valores máximos, no climatério $(5$ a 6 dias após a colheita), de aproximadamente 2.000 $\mu \mathrm{mol}$ de $\mathrm{CO}_{2} \mathrm{~kg}^{-1} \mathrm{~h}^{-1}$.

Nos frutos armazenados a $4{ }^{\circ} \mathrm{C}$, verificou-se um grande decréscimo na atividade respiratória, passando de $1.160 \mu \mathrm{mol}$ de $\mathrm{CO}_{2} \mathrm{~kg}^{-1} \mathrm{~h}^{-1}$ logo após a colheita, para aproximadamente $420 \mu \mathrm{mol}$ de $\mathrm{CO}_{2} \mathrm{~kg}^{-1} \mathrm{~h}^{-1}$ na segunda semana de armazenamento, mantendo-se posteriormente constante, entre 200 e $300 \mu \mathrm{mol} \mathrm{de} \mathrm{CO}_{2} \mathrm{~kg}^{-1} \mathrm{~h}^{-1}$, não sendo observado um pico climatérico (Figura 1B).

A produção de etileno a $23{ }^{\circ} \mathrm{C}$ aumentou a par- tir da colheita, atingindo valores máximos entre o $7^{\circ}$ e o $10^{\circ}$ dia de armazenamento, de aproximadamente $750 \mu \mathrm{mol} \mathrm{de} \mathrm{C}_{2} \mathrm{H}_{4} \mathrm{~kg}^{-1} \mathrm{~h}^{-1}$, seguido de decréscimo até valores próximos a $170 \mu \mathrm{mol}$ de $\mathrm{C}_{2} \mathrm{H}_{4} \mathrm{~kg}^{-1} \mathrm{~h}^{-1}$ (Figura 1C).

Assim como a respiração, a produção de etileno dos frutos armazenados a $4{ }^{\circ} \mathrm{C}$ manteve-se significativamente inferior à de frutos armazenados a $23{ }^{\circ} \mathrm{C}$ (Figuras $1 \mathrm{C}$ e 1D). Em frutos armazenados a $4{ }^{\circ} \mathrm{C}$, a produção de etileno foi máxima na segunda semana (378 $\mu$ mol de $\left.\mathrm{C}_{2} \mathrm{H}_{4} \mathrm{~kg}^{-1} \mathrm{~h}^{-1}\right)$ e mínima (60 $\mu$ mol de $\mathrm{C}_{2} \mathrm{H}_{4} \mathrm{~kg}^{-1} \mathrm{~h}^{-1}$ ) na quarta semana Figura 1D). A goiaba serrana possui alta produção de etileno comparativamente a outros frutos climatéricos. A produção de etileno em frutos de goiaba comum, por exemplo, é de 0,5 a 0,8 $\mu \mathrm{mol}$ de $\mathrm{C}_{2} \mathrm{H}_{4}$ $\mathrm{kg}^{-1} \mathrm{~h}^{-1}$, a $25^{\circ} \mathrm{C}$ (SINGH; PAL, 2008).

Os teores de $\mathrm{SS}$ diminuíram de $13^{\circ} \mathrm{Brix}$ para aproximadamente $11,5^{\circ}$ Brix nos frutos mantidos a $23{ }^{\circ} \mathrm{C}$ por 15 dias (Figura $1 \mathrm{E}$ ), mas, se mantiveram próximos a $13{ }^{\circ}$ Brix, mesmo depois de 30 dias, quando armazenados a $4{ }^{\circ} \mathrm{C}$ (Figura $\left.1 \mathrm{~F}\right)$. Este valor é bem superior aos valores observados em goiaba comum, que são de 7,5 a $8,5^{\circ}$ Brix para a cultivar 'Paluma', e de 6,0 a 6,7 ${ }^{\circ}$ Brix para a cultivar ' $\mathrm{Ku}$ magai' (CAVALINI et al., 2006).

Assim como o teor de SS, a AT dos frutos armazenados a $23{ }^{\circ} \mathrm{C}$ diminuiu bastante com o amadurecimento, passando de $0,68 \%$ na colheita para $0,30 \%$ após 15 dias (Figura $1 \mathrm{G}$ ). A AT também diminuiu nos frutos armazenados a $4{ }^{\circ} \mathrm{C}$, mas permaneceu acima de $0,42 \%$ da segunda a quarta semanas de armazenagem (Figura $1 \mathrm{H}$ ).

$\mathrm{O}$ maior decrécimo nos teores de $\mathrm{SS}$ e AT em goiabas serranas a $23{ }^{\circ} \mathrm{C}$, em relação a frutos armazenados a $4{ }^{\circ} \mathrm{C}$, também foi relatado por Thorp e Bieleski (2002) e, possivelmente, está relacionado à maior respiração e produção de etileno (Figuras $1 \mathrm{~A}$ a 1D). Em várias cultivares de goiaba comum, observa-se maior queda na AT dos frutos após o climatério respiratório (MERCADO-SILVA et al., 1998).

Em relação à cor da epiderme, observou-se diminuição do $h^{o}$, representando perda de cor verde, sendo maior em frutos armazenados a $23{ }^{\circ} \mathrm{C}$. Nos frutos armazenados a $23^{\circ} \mathrm{C}$, os valores de $h^{\circ}$, aos 15 dias, foram próximos a 110 (Figura 1I), sendo que, em frutos armazenados a $4{ }^{\circ} \mathrm{C}$, ao final de quatro semanas, foram próximos a 115 (Figura $1 \mathrm{~J}$ ). $\mathrm{O}$ aumento na temperatura promove a degradação da clorofila e a redução do $h^{\circ}$ da epiderme em goiaba serrana (AMARANTE et al., 2008). Todavia, em algumas cultivares de goiaba serrana, não é possível observar variações expressivas na cor da epiderme durante 
o amadurecimento dos frutos (EAST et al., 2009).

A rápida deterioração da qualidade da goiaba serrana após a colheita foi ocasionada pelo desenvolvimento acentuado de três distúrbios: 1) manchas escuras superficiais na epiderme, acompanhadas de inúmeros pontos escuros (mancha superficial); 2) manchas escuras levemente deprimidas na epiderme, principalmente na região mediana dos frutos (mancha deprimida); e 3) escurecimento da polpa. Não foi constatada a presença de patógenos nas manchas escuras superficiais e levemente deprimidas na epiderme, mostrando que estes sintomas correspondem a distúrbio fisiológico.

O escurecimento da polpa aumentou acentuadamente, tanto nos frutos armazenados a $23{ }^{\circ} \mathrm{C}$ quanto nos frutos armazenados a $4{ }^{\circ} \mathrm{C}$ (Figuras $2 \mathrm{~A}$ e 2B). Este distúrbio pode ser ocasionado principalmente pelo aumento da atividade da enzima polifenoloxidase (PPO) ou pela degradação dos açúcares no fruto, e intensificado por altas temperaturas e baixa umidade relativa durante o armazenamento (THORP; BIELESKI, 2002).

O escurecimento da polpa também pode desenvolver-se após o corte transversal do fruto e a longa exposição ao oxigênio $\left(\mathrm{O}_{2}\right)$, pela atividade da enzima PPO (THORP; BIELESKI, 2002). Em alguns casos, o escurecimento da polpa em goiaba serrana pode ser causado pela excessiva redução nos níveis de $\mathrm{O}_{2}$ e/ou aumento nos níveis de $\mathrm{CO}_{2}$ no ambiente de armazenamento (EAST et al., 2009), bem como resultado de longos períodos de armazenamento a $4{ }^{\circ} \mathrm{C}$, ocasionando assim dano de frio (THORP; BIELESKI, 2002). No presente trabalho, os frutos foram armazenados sob atmosfera do ar $\left(21 \%\right.$ de $\mathrm{O}_{2} \mathrm{e}<0,04 \%$ de $\left.\mathrm{CO}_{2}\right)$, e a análise do escurecimento da polpa foi avaliada em menos de 30 segundos após o corte dos frutos. Assim, o alto índice de escurecimento da polpa, após 15 dias a 23 ${ }^{\circ} \mathrm{C}$, pode estar relacionado à senescência dos frutos, enquanto o alto índice de escurecimento da polpa, após 30 dias a $4^{\circ} \mathrm{C}$, pode estar relacionado à senescência dos frutos e/ou à expressão de dano por frio.

$\mathrm{O}$ índice de manchas superficiais na epiderme aumentou de forma acentuada e linear nos frutos mantidos a $23^{\circ} \mathrm{C}$ (Figura 2C). Já nos frutos armazenados a $4{ }^{\circ} \mathrm{C}$, o aumento acentuado desse distúrbio ocorreu principalmente após 15 dias (Figura 2D).

Pequeno índice de manchas deprimidas na epiderme foram observadas nos frutos mantidos a $23{ }^{\circ} \mathrm{C}$, depois de três dias (Figura 2E). Aumento acentuado da incidência e severidade de manchas deprimidas foi observado nos frutos mantidos a 23 ${ }^{\circ} \mathrm{C}$, a partir do $12^{\circ}$ dia. Em frutos armazenados a $4^{\circ} \mathrm{C}$, houve aumento acentuado do índice desse distúrbio depois de 21 dias (Figura 2F).

Os índices de manchas superficiais e manchas deprimidas na epiderme dos frutos armazenados a 23 ${ }^{\circ} \mathrm{C}$, por 15 dias, foram maiores $(\mathrm{p}<0,05)$ do que aqueles em frutos armazenados a $4^{\circ} \mathrm{C}$ pelo mesmo período.

As manchas superficiais e deprimidas observadas em goiaba serrana produzidas no Brasil são semelhantes às manchas observadas em goiaba serrana produzida na Nova Zelândia (THORP; BIELESKI, 2002). Aparentemente, esses distúrbios podem ser causados por danos mecânicos durante colheita e manuseio pós-colheita, mas que se expressam durante o amadurecimento e senescência (THORP; BIELESKI, 2002).

O índice de incidência e severidade de podridões também aumentou acentuadamente e de forma linear nos frutos mantidos a $23{ }^{\circ} \mathrm{C}$, após a colheita (Figura 2G), mas manteve-se bastante baixo nos frutos armazenados a $4^{\circ} \mathrm{C}$, mesmo depois de 30 dias (Figura $2 \mathrm{H})$. Importante notar que o aumento do índice de podridões foi associado à redução significativa da $\mathrm{AT}$ dos frutos armazenados a $23{ }^{\circ} \mathrm{C}$ (Figura $1 \mathrm{G}$ ).

As podridões apresentaram-se inicialmente como pequenos pontos escuros, principalmente na região do cálice, e com o decorrer do tempo formaram extensas áreas escuras. Azzolini et al. (2005), trabalhando com goiaba 'Pedro Sato', observaram que, com o avanço do amadurecimento, o fruto tornase mais suscetível ao ataque de patógenos devido à diminuição da resistência da casca e da polpa. De acordo com East et al. (2009), as podridões em frutos de goiabeira serrana normalmente se desenvolvem após a remoção dos frutos da câmara de armazenamento refrigerado, quando os mesmos são mantidos à temperatura ambiente.

O presente estudo demonstrou que o potencial de armazenagem de goiabas serranas a $23{ }^{\circ} \mathrm{C}$ pode ser inferior a uma semana e é limitado principalmente pelo desenvolvimento dos distúrbios de escurecimento da polpa e manchas da epiderme, bem como podridões. Aparentemente, a ocorrência de escurecimento da polpa e de manchas na epiderme resulta do progresso do amadurecimento e senescência, considerando que o pico climatérico ocorreu com 5 dias após a colheita. O desenvolvimento de podridões também pode limitar a vida pós-colheita dos frutos mantidos a $23{ }^{\circ} \mathrm{C}$, embora a severidade de podridões tenha sido inferior à do escurecimento da polpa e de manchas da epiderme.

O armazenamento a $4{ }^{\circ} \mathrm{C}$ retardou significativamente o desenvolvimento de podridões, manchas superficiais e manchas deprimidas da epiderme, assim como retardou o decréscimo nos valores de SS e AT. No entanto, a redução da temperatura de 
armazenagem não preveniu o desenvolvimento do escurecimento da polpa. Esse resultado indica que o potencial de armazenagem de goiabas serrana a $4{ }^{\circ} \mathrm{C}$ é limitado, principalmente pelo desenvolvimento desse distúrbio.
Novos estudos serão necessários para avaliar o efeito de temperaturas entre $4{ }^{\circ} \mathrm{Ce} 10^{\circ} \mathrm{C}$ sobre o desenvolvimento de escurecimento da polpa e a relação com a qualidade sensorial da goiaba serrana.
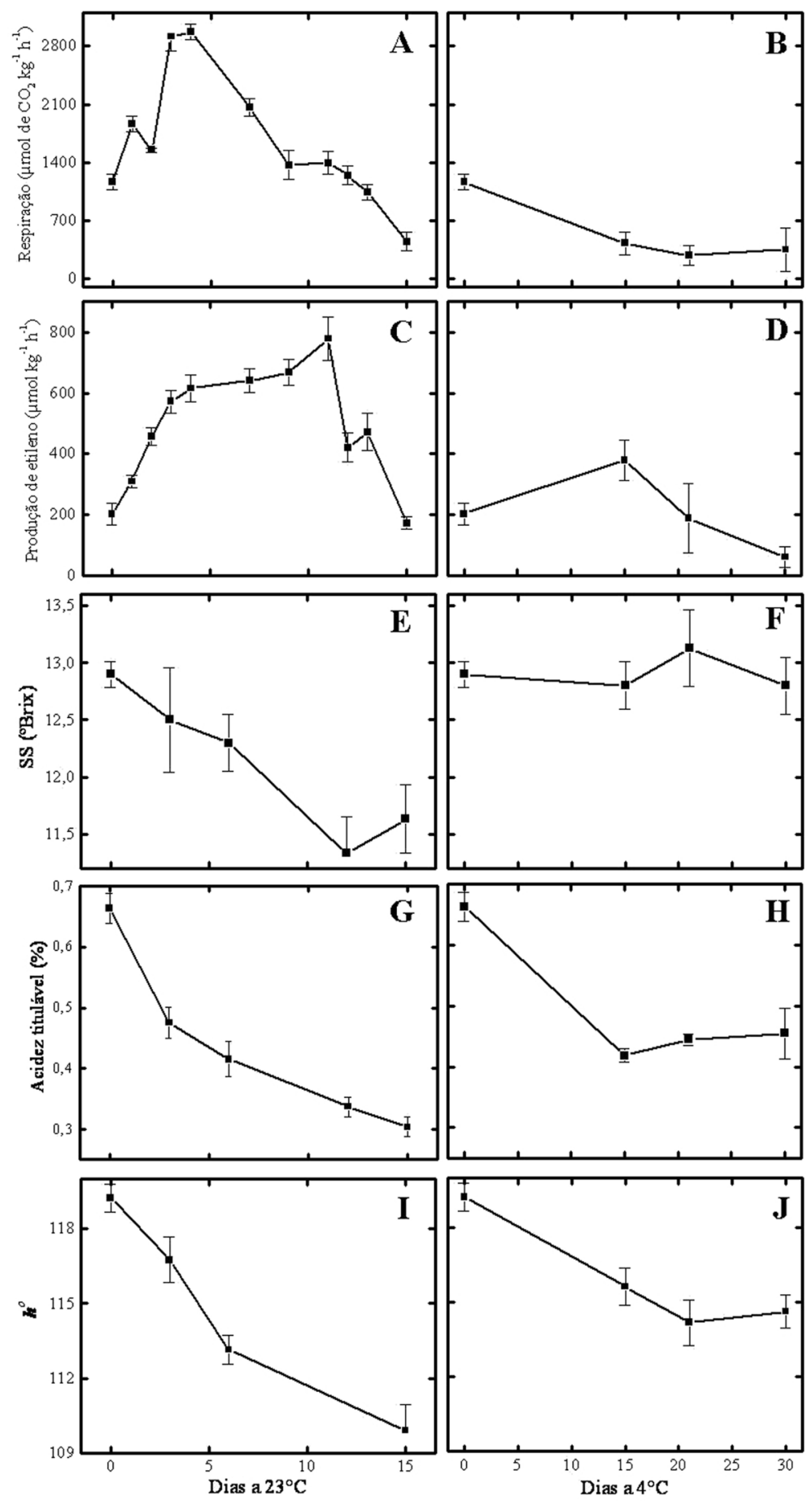

FIGURA 1 - Respiração (Ae B), produção de etileno (C e D), teor de sólidos solúveis (SS) (E e F), acidez titulável $\left(\mathrm{G}\right.$ e H) e cor da epiderme (ângulo 'hue'; $h^{\circ}$ ) (I e J), em goiaba serrana armazenada a $23{ }^{\circ} \mathrm{C}$ (gráficos à esquerda) e $4{ }^{\circ} \mathrm{C}$ (gráficos à direita). Os símbolos representam o valor médio \pm erro-padrão. 


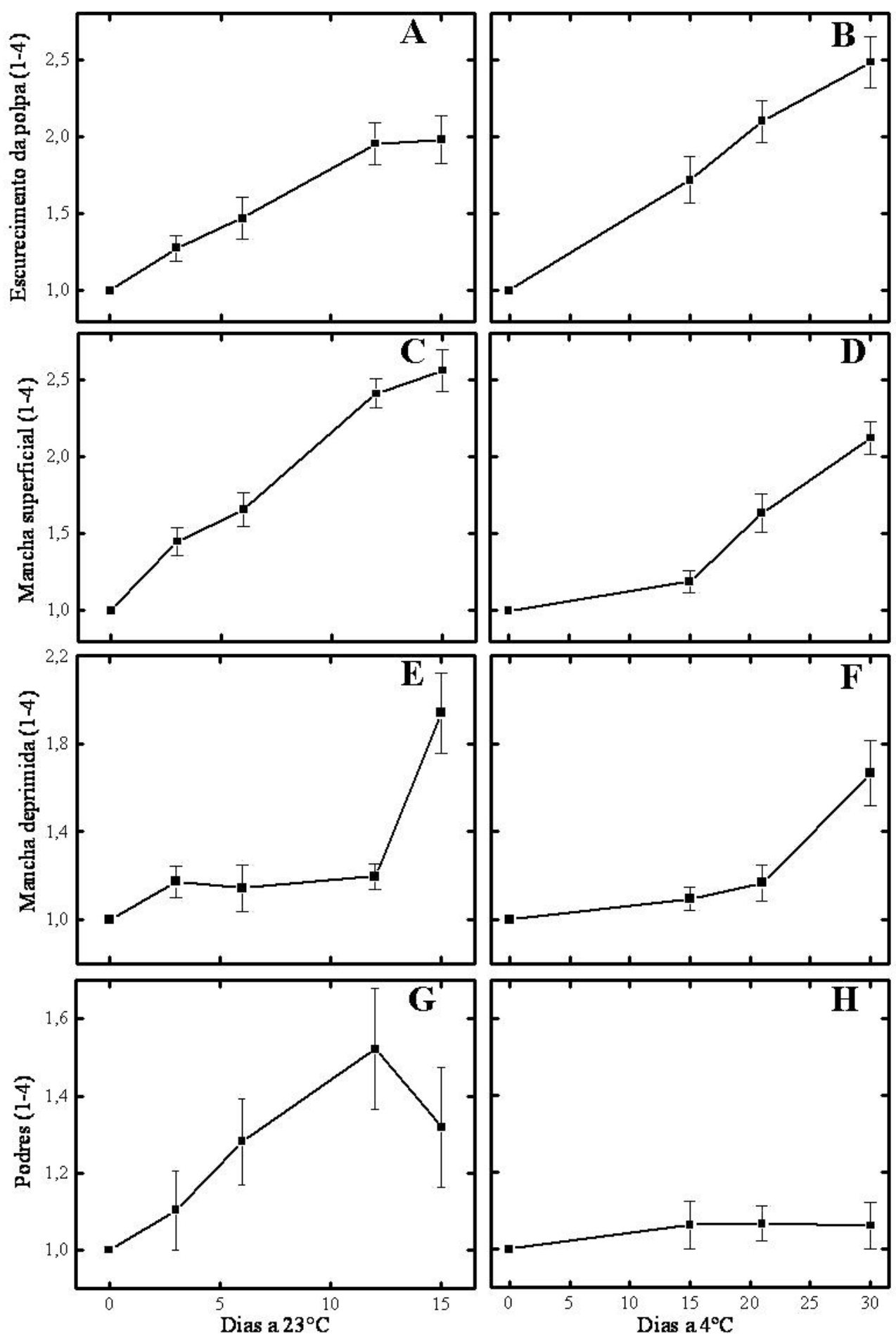

FIGURA 2 - Escurecimento da polpa (A e B), mancha superficial (C e D), mancha deprimida (E e F) e podridões $(\mathrm{G} \mathrm{e} \mathrm{H})$, em goiaba serrana armazenada a $23^{\circ} \mathrm{C}$ (gráficos à esquerda) e $4{ }^{\circ} \mathrm{C}$ (gráficos à direita). Valores de severidade, avaliada atribuindo-se notas de 1 a 4 , pela análise visual, sendo 1-ausente, 2-inicial, 3-moderada e 4-severa. Os símbolos representam o valor médio \pm 
erro-padrão.

\section{CONCLUSÕES}

1- O potencial de armazenagem de goiabas serranas a $23{ }^{\circ} \mathrm{C}$ é inferior a uma semana e limitado principalmente pelo desenvolvimento dos distúrbios de escurecimento da polpa e manchas na epiderme, bem como podridões.

2- $\mathrm{O}$ potencial de armazenagem a $4^{\circ} \mathrm{C}$ é inferior a trinta dias e limitado principalmente pelo escurecimento da polpa.

\section{AGRADECIMENTOS}

À Cooperativa Agrícola Sanjo, de São Joaquim-SC, pelo fornecimento dos frutos.

\section{REFERÊNCIAS}

AMARANTE, C.V.T. do; STEFFENS, C.A.; DUCROQUET, J.P.H.J.; SASSO, A. Qualidade de goiaba serrana em resposta à temperatura de armazenamento e ao tratamento com 1-metilciclopropeno. Pesquisa Agropecuária Brasileira, Brasília, v.43, n.12, p.1683-1689, 2008

ARGENTA, L.C.; KRAMMES, J.G.; MEGGUER, C.A.; AMARANTE, C.V.T.; MATTHEIS, J. Ripening and quality of 'Laetitia' plums following harvest and cold storage as affected by inhibition of ethylene action. Pesquisa Agropecuária Brasileira, Brasília, v.38, n.10, p.1139-1148, 2003.

AZZOLINI, M.; JACOMINO, A.P.; BRON, I.U.; KLUGE, R.A.; SCHAVINATO, M. Ripening of 'Pedro Sato' guava: study on its climateric or nonclimateric nature. Brazilian Journal of Plant Physiology, Rio de Janeiro, v.17, n.3, p. 299-306, 2005.

BRON, I.U.; RIBEIRO, R.V.; CAVALINI, F.C.; JACOMINO, A.P.; TREVISAN, M.J. Temperaturerelated changes in respiration and $\mathrm{Q}_{10}$ coefficient of guava. Scientia Agricola, Piracicaba, v.62, n.5, p.458-463, 2005.
CAVALINI, F.C.; JACOMINO, A.P.; LOCHOSKI, M.A.; KLUGE, R.A.; ORTEGA, E.M.M. Maturity indexes for 'Kumagai' and 'Paluma' guavas. Revista Brasileira de Fruticultura, Jaboticabal, v.28, n.2, p.176-179, 2006.

DUCROQUET, J.P.H.J.; HICKEL, E.R. Fenologia da goiabeira serrana (Feijoa sellowiana, Berg) no Alto Vale do Rio do Peixe, Santa Catarina. Revista Brasileira de Fruticultura, Jaboticabal, v.13, n.3, p.313-320, 1991.

EAST, A.R.; TREJO-ARAYA, X.I.; HERTOG, M.L.A.T.M.; NICHOLSON, S.E.; MAWSON, A.J. The effect of controlled atmospheres on respiration and rate of quality change in 'Unique' feijoa fruit. Postharvest Biology and Technology, Amsterdam, v.53, n.1, p.66-71, 2009.

MERCADO-SILVA, E.; BAUTISTA, P.B.; GARCIA-VELASCO, M.A. Fruit development, harvest index and ripening changes of guavas produced in Central Mexico. Postharvest Biology and Technology, Amsterdam, v.13, n.2, p.143-150, 1998.

QUADROS, K.E.; MOTA, A.P.; KERBAUY, G.B.; GUERRA, M.P.; DUCROQUET, J.P.H.J.; PESCADOR, R. Estudo anatômico do crescimento do fruto em Acca sellowiana (Berg.). Revista Brasileira de Fruticultura, Jaboticabal, v.30, n.2, p.296-302, 2008.

SINGH, S.P.; PAL, R.K. Controlled atmosphere storage of guava (Psidium guajava L.) fruit. Postharvest Biology and Technology, Amsterdam, v.47, n.3, p.296-306, 2008.

THORP, T.G.; BIELESKI, R. Feijoas: origins, cultivation and uses. Auckland: David Bateman, 2002. 87 p.

WESTON, R.J. Bioactive products from fruit of the feijoa (Feijoa sellowiana, Myrtaceae): A review. Food Chemistry, Maryland Heights, v.121, n.1, p.923-926, 2010. 